\title{
Search for MSSM Higgses at the Tevatron
}

\author{
A. Connolly ${ }^{a *}$ \\ For the CDF and D0 Collaborations

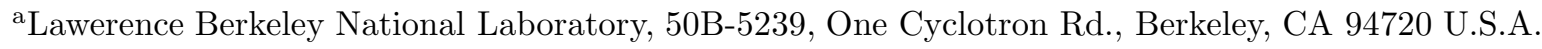

\begin{abstract}
We present an overview of searches for MSSM Higgs at the Tevatron, concentrating on searches probing the high $\tan \beta$ region. We discuss the search for $A / H \rightarrow \tau \tau$ which is soon to be completed in the Run I data and review the new tau triggers implemented by CDF and D0 in Run II, which will greatly impact this analysis. We also present the results of a Run I search for $A / H b b \rightarrow b b b b$ performed by CDF and highlight expected improvements in this channel by both experiments in Run II.
\end{abstract}

\section{MOTIVATION}

The Higgs mechanism breaks electroweak symmetry in the Standard Model, giving mass to particles through its couplings. Current data from electroweak precision measurements points to a light Higgs $\left(M_{\text {Higgs }}<199 \mathrm{GeV} @ 95 \% \mathrm{CL}\right.$ [1]). However, the Higgs has never been definitively observed $\left(M_{\text {Higgs }}>114 \mathrm{GeV}\right.$ at $\left.95 \% \mathrm{CL}[2]\right)$.

A Standard Model Higgs suffers from the socalled hierarchy problem. The theory needs finetuned parameters to accomodate a light Higgs mass. Supersymmetry offers a solution to this problem, through a symmetry between fermions and bosons.

The Minimal Supersymmetric Standard Model (MSSM) contains two Higgs doublets, leading to five physical Higgs bosons: Two neutral CP-even states (h and $\mathrm{H}$ ), one neutral $\mathrm{CP}$-odd (A), and two charged states $\left(H^{+}\right.$and $\left.H^{-}\right)$. At tree-level, the masses are governed by two parameters, often taken to be $m_{A}$ and $\tan \beta$ [3]. When $\tan \beta>>$ 1 , A is nearly degenerate with one of the CPeven states (denoted $\phi$ ). Where $m_{A} \leq 130 \mathrm{GeV}$ $\left(m_{A} \geq 130\right), m_{A} \cong m_{h}\left(m_{A} \cong m_{H}\right)$.

In this same large $\tan \beta$ region, the cross sections for some production mechanisms such as $p p \rightarrow A(\phi)$ and $p p \rightarrow A(\phi) b \bar{b}$ are enhanced by factors of $\tan \beta^{2}\left(\sec \beta^{2}\right)$. For example, with $\sqrt{s}=2$ $\mathrm{TeV}, \tan \beta=30$ and $m_{A}=100 \mathrm{GeV}$, the cross

* Current address is Fermi National Accelerator Laboratory, P.O. Box 500, M.S. 318, Batavia, IL 60510 U.S.A. sections for $p p \rightarrow A$ and $p p \rightarrow \phi$ are each of order $10 \mathrm{pb}[4]$. The cross section for $p p \rightarrow A / \phi b \bar{b}$ is smaller, but within the same order of magnitude. In the same region, the branching ratios to $A / \phi \rightarrow b \bar{b}$ and $\tau \tau$ dominate, at $\sim 90 \%$ and $\sim 10 \%$ respectively, independent of mass.

Due to their similar masses, cross-sections and branching ratios in the high $\tan \beta$ region, we search for both $\mathrm{A}$ and $\phi$ simultaneously. At the Tevatron, we search for $p p \rightarrow A / \phi \rightarrow \tau \tau$ (the $b \bar{b}$ final state is expected to be overwhelmed by dijet background) and $p p \rightarrow A / \phi b \bar{b} \rightarrow b \bar{b} b \bar{b}$.

\section{2. $\operatorname{SEARCH~FOR~} p p \rightarrow A / \phi \rightarrow \tau^{+} \tau^{-}$}

This search is underway at CDF. The dominant issues for this analysis are: tau identification, ditau mass reconstruction, irreducible background from $Z \rightarrow \tau \tau$, and event loss at the trigger level.

Wherever not specified, we use the benchmark case of $m_{A}=95 \mathrm{GeV}$ and $\tan \beta=40$ to quote efficiencies and cross-sections.

\subsection{Tau Identification}

Compared to QCD jets, taus are highly collimated, leaving narrow jets with low track and photon multiplicity, and low mass.

In $\mathrm{CDF}$, when selecting taus, one typically requires a jet with high visible $E_{T}$ containing a high $p_{T}$ track. The jet is required to be isolated in a $10^{\circ}-30^{\circ}$ annulus around the high $p_{T}$ track. The visible energy in a $10^{\circ}$ cone is required to satisfy low track and photon multiplicity requirements 
and to reconstruct a mass $m<1.8 \mathrm{GeV}$. Additionally, a requirement is made on the charge of the tracks in the $10^{\circ}$ cone when appropriate. In Run I, CDF acheived fake rates in the range 1.2 - $0.7 \%$ for jet $E_{T}$ between 20 and $200 \mathrm{GeV}[5]$.

\subsection{Ditau Mass Reconstruction}

The full mass of a ditau system may be reconstructed [6] if the neutrinos are assumed to travel in the same direction as their parent taus, by solving the following system of equations:

$$
\begin{aligned}
& \not_{x}^{\text {meas }}=\not_{x}^{\tau 1}+\not_{x}^{\tau 2} \\
& \not_{y}^{\text {meas }}=\not_{y}^{\tau 1}+\not_{y}^{\tau 2}
\end{aligned}
$$

where $\mathbb{F}_{x, y}^{\text {meas }}$ are the $\mathrm{x}$ and $\mathrm{y}$ components of the measured event missing energy, and $\mathbb{E}_{x, y}^{\tau 1}$ and $\mathbb{E}_{x, y}^{\tau 2}$ denote the missing energy from each tau.

Equations 11 and 2 do not give a meaningful solution when the taus are back-to-back in the transverse plane. Therefore, we require that $|\sin \Delta \phi|>0.3$, where $\Delta \phi$ is the azimuthal angle between the tau candidates.

When the solution to Equations 11 and 2 gives $H^{\tau 1}<0$ or $H^{\tau 2}<0$, the event is thrown out, causing about $50 \%$ of the Higgs signal to be lost. However, $97 \%$ of $\mathrm{W}+$ jets events are rejected in this way, which would otherwise be a formidable background.

We generate $A / \phi \rightarrow \tau \tau$ events in Pythia 6.203 with $m_{A}=95 \mathrm{GeV}$ and $\tan \beta=40$. After simulation of the Run I CDF detector, a ditau mass distribution is reconstructed with a mean value of $93.7 \mathrm{GeV}$ with an RMS of $24.1 \mathrm{GeV}$.

\subsection{Irreducible Background}

The dominant reducible backgrounds to this analysis are $\mathrm{QCD}, Z \rightarrow e e$, and $\mathrm{W}+$ jets. $Z \rightarrow \tau \tau$ is an irreducible background, but Higgs events are more efficient for this search than Z's for a couple of reasons.

First, in the high $\tan \beta$ region, $A / \phi$ 's have a high branching ratio to taus (9\%) compared to $Z$ 's $(3.7 \%)$. Second, an $A / \phi$ is typically produced with a stiffer $p_{T}$ than a $Z$. This means that the requirement $|\sin \Delta \phi|>0.3$, which is nearly equivalent to $p_{T}^{A / \phi / Z}>15 \mathrm{GeV}$, is $\sim 30 \%$ more efficient for Higgs events than $\mathrm{Z}$ events.

\subsection{Triggers}

Since there was no $\tau$ trigger in Run I at CDF, the analysis uses a lepton trigger requiring $p_{T}>$ $18 \mathrm{GeV}$, seeking events with one leptonic and one hadronic decay. Since only half of the signal events decay in this way, and of these, only $20 \%$ contain a lepton which satisfies the $p_{T}$ requirement within the acceptance, the signal rate is greatly diminished at the trigger level.

This major loss at the trigger level is problematic, since the cross section drops by a factor of 4 from $m_{A}=95 \mathrm{GeV}$ to $m_{A}=120 \mathrm{GeV}$, before the mass reconstruction, with an RMS of $24 \mathrm{GeV}$, can discriminate from $Z \rightarrow \tau \tau$. Therefore, in Run II, CDF and D0 are both implementing triggers designed for tau physics. Lowered $p_{T}$ thresholds and new decay modes available will greatly increase the acceptance for this search.

In Run II, CDF and D0 both have lepton + track triggers and $\tau+\mathbb{E}_{T}$ triggers. In addition, both experiments are triggering on events with two hadronic taus. CDF's trigger is calorimeterbased, while D0's is track-based.

The Run I search for $A / \phi \rightarrow \tau \tau$ is still work in progress, and the Run II analysis is also in the works.

\section{SEARCH FOR $p p \rightarrow A / \phi b \bar{b} \rightarrow b \bar{b} b \bar{b}$}

CDF performed this search in Run I. Both experiments expect to improve on the analysis in Run II.

\subsection{Run I search}

The Run I search at CDF [7] utilized a 4-jet trigger requiring $\Sigma E_{T}>125 \mathrm{GeV}$. Three b-tags were required based on displaced vertices, and the $\mathrm{b}$ jets were required to be separated in azimuthal angle, $\Delta \phi>1.9$. To optimize sensitivity, the $E_{T}$ cuts on the jets varied with mass hypothesis. For mass hypothesis below $120 \mathrm{GeV}$ (above $120 \mathrm{GeV}$ ), the second and third b-tagged jets (first and second jets) ordered in $E_{T}$ were chosen for the mass reconstruction. The search is performed in mass windows dependedent on mass hypothesis.

The product of branching ratio and acceptance ranged from 0.2 to $0.6 \%$ in the mass range 70 and $300 \mathrm{GeV}$. For a mass hypothesis of $70 \mathrm{GeV}$, 
5 events were observed with $4.6 \pm 1.4$ expected. Only these 5 events appear in the higher mass windows. No excess above predicted is observed. Figure 1 shows the $m_{A}-\tan \beta$ region excluded.

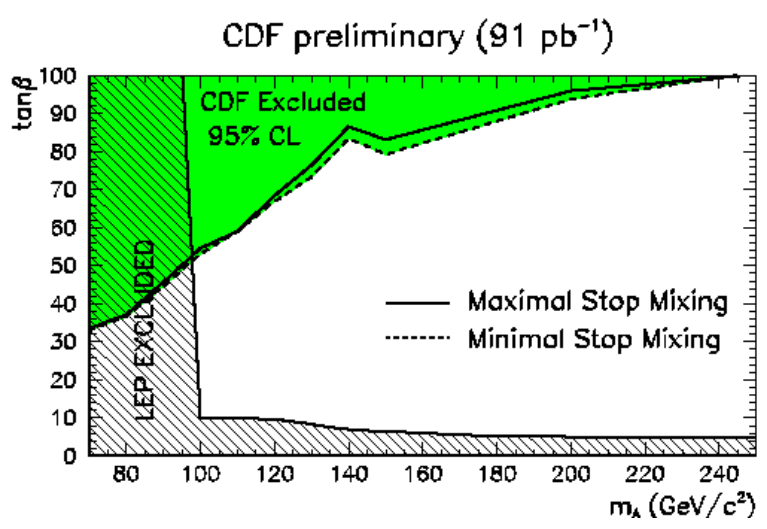

Figure 1. Region of the $m_{A}-\tan \beta$ region exluded by the CDF search.

\subsection{Run II Improvements}

At $\mathrm{CDF}$, studies of $Z \rightarrow b \bar{b}$ events show an improved dijet mass resolution after correcting for muons, $E_{T}$, and nonlinearities in the hadronic calorimeter. Separate studies of QCD jets using similar techniques show a $30 \%$ improvement in jet resolution.

B-tagging in Run II at CDF will be improved with the new ability to reconstruct threedimensional tracks. Extended coverage from $|\eta|<1$ (Run I) to $|\eta|<2$ means improve b-jet and lepton acceptance. Additionally, new triggers will also recover acceptance, including a displaced track trigger, and an improved multijet trigger.

With a new silicon detector, D0 will also be performing this search in Run II, expecting a $12 \%$ dijet mass resolution. Both experiments perform a study of their expected sensitivity to $p p \rightarrow A / \phi b \bar{b} \rightarrow b \bar{b} b \bar{b}$ in Run II, and obtain similar results $[8]$. We present the D0 study here.

D0 also uses a multijet trigger, requiring four jets, each with $E_{T}>15 \mathrm{GeV}$. To maximize sensitivity, mass dependent $E_{T}$ cuts are made on the jets. At least $3 \mathrm{~b}$ tags are required. All mass combinations are plotted, and a $2.5 \sigma b \bar{b}$ mass window is used. With $2 f b^{-1}$, D0 concludes that the Tevatron is expected to exclude $m_{A}<160 \mathrm{GeV}$ for $\tan \beta=40$ at $95 \% \mathrm{CL}$, and a $5 \sigma$ discovery for $m_{A}<115 \mathrm{GeV}$ for the same $\tan \beta$.

\section{CONCLUSIONS}

Run I results of the search for $A / \phi \rightarrow \tau \tau$ at $\mathrm{CDF}$ are to be completed soon, and a first glimpse of Run II data is on the way.

The Run I search for $p p \rightarrow A / \phi b \bar{b} \rightarrow b \bar{b} b \bar{b}$ derives lower mass limits for $\tan \beta$ in excess of 35 . In Run II with both experiments searching for this decay mode, the Tevatron is expected to exclude (or make a discovery in) a significant region of MSSM parameter space. Both experiments are optimistic about improvements from triggers, jet resolution, and b-tagging to make this search even stronger than the current projections.

\section{REFERENCES}

1. K. Hagiwara et al., Physical Review D66, 010001 (2002).

2. ALEPH Collaboration, DELPHI Collaboration, L3 Collaboration, OPAL Collaboration and LEP Higgs Working Group, LHWG Note/2001-03, hep-ex/0107029.

3. For a review of the MSSM, see H.P. Nilles, Phys. Rept. 110:1,1984, H. Haber, G. Kane, Phys. Rept. 117:75,1985.

4. M. Spira, Nucl.Instrum.Meth.A389:357360,1997

5. CDF Collaboration, Phys.Rev.Lett.79:357362, 1997

6. CMS Technical Proposal, CERN/LHCC 9438, December 1994, pp. 191-192.

7. CDF Collaboration, Phys.Rev.Lett.86(2001) 4472-4478.

8. M.Carena, J.S.Conway, H.E.Haber, J.D.Hobbs, et al, Fermilab-Conf-00/279$\mathrm{T}$ and SCIPP-00/37 hep-ph/0010338. 